\title{
Correlation of Hepatic Steatosis with Hepatic Fibrosis in NAFLD Patients by Fibroscan
}

\author{
Rishabh Sehgal ${ }^{\circledR 1}$, Jyotisterna Mittal ${ }^{\circledR 2}$, Inderpal Singh ${ }^{\circledR 3}$ \\ ${ }^{1}$ Senior Resident, Department of Gastroentrology, Dayanand Medical College and Hospital, Ludhiana, Punjab, India, ${ }^{2}$ Consultant Dermatologist, Vijay Anand hospital, \\ Ludhiana, Punjab, India, ${ }^{3}$ Senior Resident, Department of Medicine, Government Medical College, Patiala, Punjab, India.
}

\section{Abstract}

Introduction: Nonalcoholic fatty liver disease (NAFLD) is a significant cause of liver injury in the world. Transient elastography with controlled attenuation parameter (CAP) is now days commonly used as a non-invasive modality to quantify liver steatosis and stage of Fibrosis in the Liver. This study was done to the correlation of hepatic Steatosis with hepatic Fibrosis in NAFLD Patients by fibroscan. Subjects and Methods: All NAFLD patients coming to DMCH from 1/1/18 to 30/11/18 were retrospectively analysed for the presence of any correlation between Steatosis and Fibrosis using a controlled attenuation parameter (CAP) and liver stiffness measurement (kPa), respectively by Fibroscan. Patients with a history of significant alcohol intake, viral infection, severe weight loss, on TPN, on drugs like amiodarone, diltiazem, steroids were excluded. Along with this history of hypertension, diabetes and smoking were noted from the available data. Results: The mean CAP of all 446 patients was $310.58 \pm 53.55$ and the mean $\mathrm{kPa}$ was $7.14 \pm 4.75$. Overall there was a significant correlation between CAP and kPa in all NAFLD patients $(\mathrm{p}<0.000)$. This was also true in patients who were more than 20 years of age, who have increased levels of triglycerides and were obese. Patients with S0 steatosis had a mean $\mathrm{kPa}$ value of 5.33 and as the steatosis stage worsened to S3 mean $\mathrm{kPa}$ value also increased to a maximum of 7.63 . Conclusion: Quantification of Steatosis by CAP has a significant correlation with the stage of Fibrosis, especially in patients with increasing age, obese and who have high triglyceride levels.

Keywords: Fibroscan, Controlled Attenuation Parameter, Non Alcohlic Fatty Liver Disease, Hepatic Steatosis, Liver Stiffness Measurement

Corresponding Author: Inderpal Singh, Senior Resident, Department of Medicine, Government Medical College, Patiala, Punjab, India. E-mail: drinderpal@gmail.com

Received: 11 August 2020

\section{Introduction}

Non-alcoholic fatty liver disease (NAFLD) is now the commonest cause of chronic liver disease worldwide. It causes serious hepatic injury and can lead to Fibrosis, cirrhosis, and hepatocellular carcinoma. Worldwide NAFLD is prevalent in $24 \%-25 \%$ of the general population, ${ }^{[1]}$ whereas its prevalence in India is $9-32 \% .{ }^{[2]}$ The prevalence of NAFLD ranges from $22.5 \%$ to $44 \%$ in children with obesity. ${ }^{[3]}$

The pathogenesis of NAFLD is still unclear. There are multiple theories. According to the famous " Two Hit theory," its natural history includes 2 phases. In the first phase, there is an accumulation of triglycerides due to insulin resistance leading to Steatosis, and in the second phase, oxidative stress causes a hit on the vulnerable liver leading to inflammation and Fibrosis. ${ }^{[4]}$ Then there is a Multiple parallel-hits theory according to which the "first hit" is a sum of multiple factors that brings down liver defenses. ${ }^{[5]}$ Another theory called the Distinct-hit theory describes NAFLD and NASH as two separate entities, which are associated with insulin resistance but are not related to each other. ${ }^{[6]}$

Whether triglycerides leading to Steatosis are by themselves toxic for the liver or are just innocent bystanders is still uncertain. A study showed a significant correlation between severe Fibrosis and severe Steatosis associated with hypertriglyceridemia and waist circumference in NAFLD patients. ${ }^{[7]}$ On the other hand, studies are suggesting that the accumulation of triglycerides may be a defense mechanism against liver injury, thus causing an inverse relation between Steatosis and Fibrosis. ${ }^{[8,9]}$

Currently, the routinely used modalities like laboratory tests and ultrasonography are unable to determine the levels of Steatosis and Fibrosis adequately or cannot be applied as a screening procedure (liver biopsy). Among the noninvasive tests, transient elastography with controlled attenuation parameter (CAP) is very accurate in quantifying liver steatosis and staging fibrosis in patients with NAFLD. The 
method is fast, reproducible and reliable, thus allowing for population-wide screening and disease follow-up. There is a dearth of data describing the relationship between the severity of hepatic Steatosis and the severity of Fibrosis, hence this study was conducted to find a correlation between the grade of Steatosis and stage of Fibrosis using Fibroscan in patients with NAFLD.

\section{Subjects and Methods}

All NAFLD patients coming to DMCH from $1 / 1 / 18$ to $30 / 11 / 18$ were retrospectively analysed for the presence of any correlation between Steatosis and Fibrosis using controlled attenuation parameter (CAP) and liver stiffness measurement $(\mathrm{kPa})$, respectively by Fibroscan. Patients with a history of significant alcohol intake, viral infection, severe weight loss, on TPN, on drugs like amiodarone, diltiazem, steroids were excluded. Along with this history of hypertension, diabetes and smoking were noted from the available data.

\section{Liver stiffness measurement:}

Liver stiffness was measured using signals acquired by the Fibro Scan M probe or XL probe based on vibration-controlled elastography. The patient lied in dorsal decubitus position with the right arm in maximal abduction and the operator located a liver portion on the right lobe of the liver which is devoid of large vascular structures using time-motion ultrasound image. When the target area had been located, the $\mathrm{M}$ probe/X probe button was pressed to start the measurements. The final result was the median value of ten measurements performed between 25 and $65 \mathrm{~mm}$ depth and was expressed in $\mathrm{kPa}$. Procedures with at least ten valid shots and interquartile range (IQR) inferior to $30 \%$ were considered reliable. All measurements were performed by the same operator. The threshold used $(8.7 \mathrm{kPa})$ for severe Fibrosis was the value determined in a study by Wong et al. in 2010 with a sensitivity of $83.9 \%$, the specificity of $83.2 \%$ and a negative predictive value of $94.6 \%{ }^{[10]}$

\section{Controlled attenuation parameter (CAP):}

The controlled attenuation parameter measures liver ultrasonic attenuation at $3.5 \mathrm{MHz} .{ }^{[11]}$ It was computed with the same signals as the one used to measure liver stiffness. Therefore both stiffness and CAP were obtained simultaneously in the same volume of liver parenchyma. The final CAP value was the median value of the ten individual measurements and was expressed in $\mathrm{dB} / \mathrm{m}$, [Table 1\&2].

\section{Statistical analysis:}

Variables were expressed as mean \pm standard deviations. Pearson correlation was applied to find a correlation between
Table 1: Values used for staging of Fibrosis [based on Wong et al 2010 and reference values provided with Fibroscan (Echosens)]

\begin{tabular}{ll}
\hline Stage of Fibrosis & $\mathbf{k P a}$ \\
\hline F0 & $0-6$ \\
\hline F1 & $6.1-7.2$ \\
\hline F2 & $7.3-8.2$ \\
\hline F2-3 & $8.3-8.7$ \\
\hline F3 (severe fibrosis ) & $8.8-10.5$ \\
\hline F3-4 (severe fibrosis ) & $10.6-11.8$ \\
\hline F4 (cirrhosis) & $>11.8$ \\
\hline
\end{tabular}

Table 2: Values used for grading of Steatosis [based on reference values provided with Fibroscan (Echosens)]

\begin{tabular}{ll}
\hline Grade of Steatosis & CAP $(\mathbf{d b} / \mathbf{m})$ \\
\hline S0 & $<237.7$ \\
\hline S1 & $237.7-259.4$ \\
S2 & $259.4-292.3$ \\
\hline S3 (severe steatosis ) & $>292.3$ \\
\hline
\end{tabular}

Steatosis and Fibrosis (measured by CAP and kPa respectively) and the effect of hypertriglyceridemia, waist circumference and diabetes status on this correlation was studied. Significance was defined by $\mathrm{p}<0.05$.

\section{Results}

446 patients were included in the study and out of these, $70 \%$ were males. The mean AST was $30.5 \pm 2$ and the mean ALT was $35.4 \pm 1.5$. The mean age was 46.7 years. Out of 446 patients; with the available data $55.1 \%$ of patients were obese (BMI $\geq 25$ ), $2.7 \%$ of patients were hypertensive, $19.3 \%$ of patients were diabetic and $56.7 \%$ of patients have increased triglyceride levels $(\geq 150 \mathrm{mg} / \mathrm{dl})$. Baseline characteristics of NAFLD patients. [Table 3].

The mean CAP of all patients was $310.58 \pm 53.55$ and meant $\mathrm{kPa}$ was $7.14 \pm 4.75$. It was found that those who had aged more than 20 years had higher mean CAP and mean $\mathrm{kPa}$ than those who were young. Similarily higher mean CAP and mean $\mathrm{kPa}$ was found in patients who were obese or had hypertension or diabetes or hypertriglyceridemia than those who were not obese or normotensive or non-diabetic or had normal triglycerides level respectively. This is shown in [Table 4]. Overall there was a significant correlation between CAP and $\mathrm{kPa}$ in all NAFLD patients $(\mathrm{p}<0.000)$ shown in [Figure 1 $\&$ Table 5]. This was also true in patients who were more than 20 years of age, who have increased levels of triglycerides and were obese. [Table 5]. 
It was tried to find out the percentage of patients with severe fibrosis $(\geq F 3)$ in each grade of Steatosis and it was found that as the stage of Steatosis worsens there is an increase in several patients with severe Fibrosis ( increased from $0 \%$ in S0 to $23.38 \%$ in S3). It was also found that patients with S0 steatosis had a mean $\mathrm{kPa}$ value of 5.33 and as the steatosis stage worsened to $\mathrm{S} 3$ mean $\mathrm{kPa}$ value also increased to a maximum of 7.63 as shown in [Table 6,7 \& Figure 2]. Patients with multiple components of metabolic syndrome were compared using their mean CAP and $\mathrm{kPa}$ values, but no particular pattern was observed [Table 8].

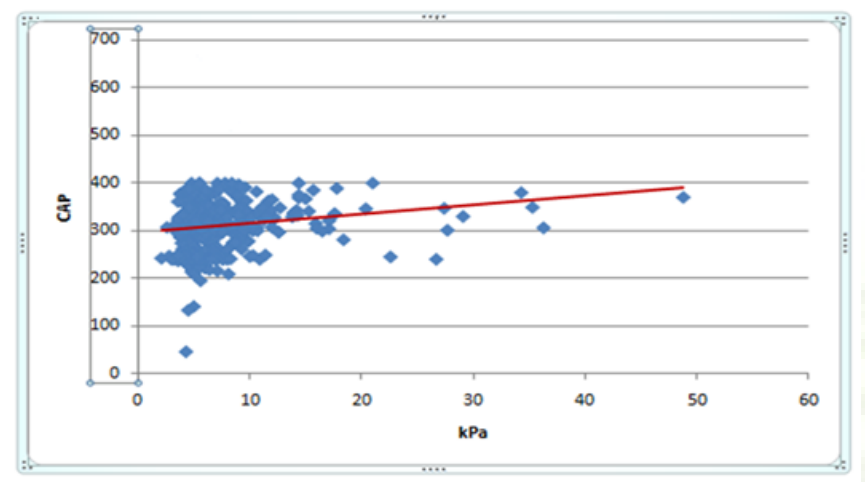

Figure 1: CAP value(y-axis) plotted against values of LSM ( $x$ axis)

Overall there was a significant correlation between CAP and $\mathrm{kPa}$ in all NAFLD patients $(\mathrm{p}<0.000)$ shown in [Figure 1].

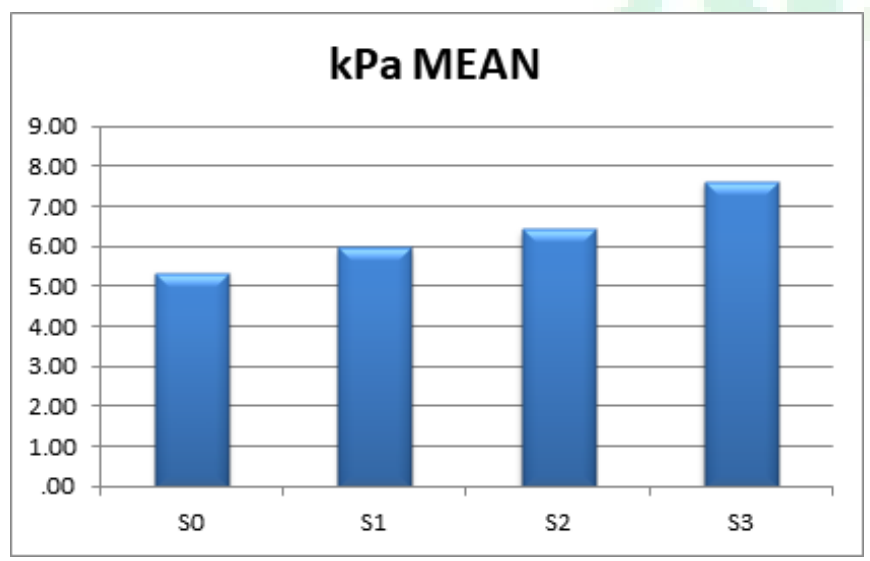

Figure 2: Mean kPa value of patients with each grade of steatosis

\section{Discussion}

Our study shows a significant correlation between Steatosis and Fibrosis, especially in patients who were more than 40 years old and have hypertriglyceridemia and obesity. This is expected as Steatosis precedes inflammation and Fibrosis in the natural history of NAFLD. ${ }^{[12]}$

This is similar to a study conducted by Marty et al. wherein a significant correlation between Steatosis and Fibrosis associated with hypertriglyceridemia and waist circumference was found in diabetic patients. ${ }^{[7]}$ Similarly, a Japanese study conducted by Cho et al found a significant correlation of Steatosis and Fibrosis in the pediatric obese group. ${ }^{[3]}$ However, two experimental studies have suggested that Steatosis protects rather than promote Fibrosis. Listenberg et al. have reported that the incorporation of fatty acids in the hepatic triglyceride pool prevented their pro-apoptotic effects. ${ }^{[8]}$ Yamagushi et al. found more inflammation and Fibrosis in mice whose hepatic triglyceride synthesis was reduced due to DGAT inhibition. ${ }^{[9]}$ If triglycerides protected the liver from Fibrosis, we should have found an inverse relation between Steatosis and Fibrosis.

In the present study, we found a high rate of severe liver fibrosis $(19.05 \%)$ and severe Steatosis (69\%) which could be expected as all patients were those who came to the hospital for a medical reason like fatigue, right hypochondria heaviness, uncontrolled diabetes etc. Studies using non-invasive methods reported severe Fibrosis in $5.6 \%$ of outpatients and up to $15 \%$ in hospitalized patients. ${ }^{[13,14]}$ As compared to non-invasive methods, in a study in which biopsy was performed on diabetic patients the rate of severe Fibrosis reached 35\%. ${ }^{[15]}$ In another study conducted by Marty et al. using Fibroscan; 41\% of diabetic patients had severe Steatosis. ${ }^{[7]}$

NAFLD and metabolic syndrome (MetS) are associated with insulin resistance (IR) is the common pathogenetic factor. The presence of MetS increases the risk of development of NAFLD and more the number of MetS components present is the risk of severe NAFLD. ${ }^{[16]}$ From the available data, we found that patients those who had higher BMI or high triglyceride levels had higher Steatosis and Fibrosis as compare to those who have normal BMI or normal triglyceride level. Similarily diabetic patients (DM) or patients with hypertension (HTN) had higher Steatosis and Fibrosis and various studies have shown similar results. ${ }^{[3,7]}$ The presence of obesity or hypertriglyceridemia was associated with a significant correlation of Steatosis and Fibrosis. However, the presence of DM or HTN was not associated with any correlation between the two, which could be because of a small number of DM and HTN patients in the present study. We also tried to analyse the relationship between the number of MetS components and the degree of Steatosis and Fibrosis, but we did not find any relation which could be because of the retrospective nature of data. 


\begin{tabular}{llll}
\hline Table 3: Baseline characteristics of NAFLD patients & & Po. of Patients \\
\hline & & 446 & - \\
\hline Total & & 312 & $70.00 \%$ \\
\hline Male & & 134 & $30.00 \%$ \\
\hline Female & & Mean $=30.5 \pm 2$ \\
\hline AST & 446 & Mean $=35.4 \pm 1.5$ \\
\hline ALT & $0-20$ & 946 & $2 \%$ \\
\hline Age & $21-40$ & 141 & $31.61 \%$ \\
& $>40$ & 296 & $66.36 \%$ \\
\hline BMI & $\leq 22.9$ & 14 & $3.1 \%$ \\
& $23-24.9$ & 38 & $8.5 \%$ \\
& $\geq 25$ & 246 & $55.1 \%$ \\
\hline HTN & Data Not Available & 148 & $33.1 \%$ \\
& Yes & 12 & $2.7 \%$ \\
& No & 304 & $68.20 \%$ \\
\hline DM & Data Not Available & 130 & $29.10 \%$ \\
& Yes & 86 & $19.30 \%$ \\
& No & 230 & $51.60 \%$ \\
\hline Triglycerides & Data Not Available & 130 & $29.10 \%$ \\
& Increased & 253 & $56.70 \%$ \\
& Normal & 27 & $6.10 \%$ \\
\hline & Data Not Available & 166 & $37.20 \%$ \\
\hline
\end{tabular}

\begin{tabular}{|c|c|c|c|c|}
\hline & & No. of Patients & Mean CAP & Mean kPa \\
\hline Total & & 446 & $310.58 \pm 53.55$ & $7.14 \pm 4.75$ \\
\hline Male & & 312 & $310.03 \pm 53.3$ & $6.98 \pm 4.31$ \\
\hline Female & & 134 & $311.86 \pm 54.31$ & $7.49 \pm 5.63$ \\
\hline \multirow[t]{3}{*}{ Age } & $0-20$ & 9 & $258.67 \pm 51.03$ & $5.89 \pm 1.34$ \\
\hline & $21-40$ & 141 & $313.26 \pm 59.49$ & $6.31 \pm 2.79$ \\
\hline & $>40$ & 296 & $310.88 \pm 49.9$ & $7.48 \pm 5.18$ \\
\hline \multirow[t]{3}{*}{ BMI } & $\leq 22.9$ & 14 & $286.14 \pm 56.61$ & $5.4 \pm 1.15$ \\
\hline & $23-24.9$ & 38 & $298.87 \pm 44.73$ & $5.37 \pm 1.05$ \\
\hline & $\geq 25$ & 246 & $310.03 \pm 50.51$ & $7.22 \pm 4.15$ \\
\hline \multirow[t]{2}{*}{ HTN } & Yes & 12 & $329 \pm 41.99$ & $7.82 \pm 2.36$ \\
\hline & No & 304 & $309.11 \pm 54.61$ & $6.94 \pm 4.2$ \\
\hline \multirow[t]{2}{*}{ DM } & Yes & 86 & $319.35 \pm 47.97$ & $8.94 \pm 6.02$ \\
\hline & No & 230 & $306.32 \pm 56.12$ & $6.24 \pm 2.87$ \\
\hline \multirow[t]{2}{*}{ Triglycerides } & Increased & 253 & $308.92 \pm 51.56$ & $7.15 \pm 4.02$ \\
\hline & Normal & 27 & $294.15 \pm 43.58$ & $5 \pm 0.9$ \\
\hline
\end{tabular}

Our study does have some limitations as it is a retrospective study, so complete data of all patients was not available. The second limitation is the lack of histological confirmation of our results. It was impossible to perform a liver biopsy in 


\begin{tabular}{llll}
\hline Table 5: Correlation of CAP and kPa & & & \\
\hline Correlation of CAP \& $\mathbf{~ k a}$ & $\mathbf{N}$ & Pearson Correlation & P-Value \\
\hline Overall & 446 & $.167^{* *}$ & .000 \\
Age 0-20 & 9 & 0.392 & .296 \\
\hline Age $21-40$ & 141 & $.252^{* *}$ & .003 \\
\hline Age more than 40 & 296 & $.164^{* *}$ & .005 \\
\hline Only Male & 312 & $.192^{* *}$ & .001 \\
\hline Only Female & 134 & 0.126 & .146 \\
\hline Normal Triglycerides & 27 & 0.185 & .355 \\
\hline Increased Triglycerides & 253 & $.175^{* *}$ & .005 \\
\hline BMI Normal & 14 & 0.049 & .867 \\
\hline BMI Overweight & 38 & -0.102 & .543 \\
\hline BMI Obese & 246 & $.183^{* *}$ & .004 \\
\hline HTN & 12 & 0.206 & .520 \\
\hline DM & 86 & 0.149 & .172 \\
\hline
\end{tabular}

Table 6: Percentage of patients with a particular stage of Fibrosis in each grade of Steatosis

\begin{tabular}{|lllllllll}
\hline S Grade & F Stage & & & & & \multicolumn{2}{c}{ Total } \\
\hline & Fo & F1 & F2 & F2-F3 & F3 & F3-F4 & F4 & \\
\hline & 16 & 2 & 1 & 0 & 0 & 0 & 0 & 19 \\
\hline S1 & $84.21 \%$ & $10.53 \%$ & $5.26 \%$ & $0.00 \%$ & $0.00 \%$ & $0.00 \%$ & $0.00 \%$ & $100.00 \%$ \\
\hline & 53 & 9 & 4 & 1 & 2 & 2 & 2 & 73 \\
\hline S2 & $72.60 \%$ & $12.33 \%$ & $5.48 \%$ & $1.37 \%$ & $2.74 \%$ & $2.74 \%$ & $2.74 \%$ & $100.00 \%$ \\
\hline & 24 & 9 & 3 & 3 & 6 & 0 & 1 & 46 \\
\hline S3 & $52.17 \%$ & $19.57 \%$ & $6.52 \%$ & $6.52 \%$ & $13.04 \%$ & $0.00 \%$ & $2.17 \%$ & $100.00 \%$ \\
\hline & 155 & 51 & 20 & 10 & 26 & 10 & 36 & 308 \\
\hline & $50.32 \%$ & $16.56 \%$ & $6.49 \%$ & $3.25 \%$ & $8.44 \%$ & $3.25 \%$ & $11.69 \%$ & $100.00 \%$ \\
\hline
\end{tabular}

Table 7: Mean kPa value of patients with each grade of Steatosis

\begin{tabular}{lllll}
\hline S Grade & kPa Mean & N & SD & P-Value \\
S0 & 5.33 & 19 & 1.05990 & .008 \\
\hline S1 & 5.96 & 73 & 3.64354 & \\
S2 & 6.42 & 46 & 2.56728 & \\
S3 & 7.63 & 308 & 5.25000 & \\
Total & 7.1368 & 446 & 4.74547 & \\
\hline
\end{tabular}

all patients as it is invasive and has its risks. However, the diagnostic performances of Fibroscan and CAP have been validated in several studies. ${ }^{[17,18]}$ The third limitation is that inflammatory markers were not analysed. However, a large number of patients (446) were analysed and that too by a single operator. Also XL probe was used for obese patients to get accurate values as the use of $\mathrm{M}$ probe had resulted in false high values in this subset of patients. ${ }^{[19]}$ We are also conducting a prospective longitudinal study to overcome some of the limitations. 


\begin{tabular}{|c|c|c|c|}
\hline $\begin{array}{l}\text { Metabolic syndrome compo- } \\
\text { nents }\end{array}$ & No. of Patients & Mean CAP & Mean kPa \\
\hline DM, HTN, Obese, Triglycerides & 5 & $325.4 \pm 36.34$ & $9.28 \pm 2.52$ \\
\hline DM, HTN, Obese & 5 & $325.4 \pm 36.34$ & $9.28 \pm 2.52$ \\
\hline DM, HTN, Triglycerides & 6 & $314.5 \pm 42.07$ & $8.8 \pm 2.54$ \\
\hline DM, Obese, Triglycerides & 58 & $324.84 \pm 46.07$ & $9.7 \pm 5.76$ \\
\hline HTN, Obese, Triglycerides & 10 & $340.4 \pm 35.45$ & $8.22 \pm 2.37$ \\
\hline DM, HTN & 6 & $314.5 \pm 42.07$ & $8.8 \pm 2.54$ \\
\hline DM, Obese & 74 & $320.88 \pm 46.27$ & $9 \pm 5.5$ \\
\hline DM, Triglycerides & 66 & $320.76 \pm 49.17$ & $9.17 \pm 5.6$ \\
\hline HTN, Obese & 10 & $340.4 \pm 35.45$ & $8.22 \pm 2.37$ \\
\hline HTN, Triglycerides & 11 & $333.09 \pm 41.46$ & $8.05 \pm 2.31$ \\
\hline Obese, Triglycerides & 211 & $311.77 \pm 51.29$ & $7.47 \pm 4.31$ \\
\hline
\end{tabular}

\section{Conclusion}

Quantification of Steatosis by CAP has a significant correlation with the stage of Fibrosis, especially in patients with increasing age, those who are obese and who have high triglyceride levels. Prevalence of NAFLD is on the rise and by taking efforts at the stage of Steatosis (reversible) like lifestyle modifications or development of drugs, we can prevent Fibrosis which is largely irreversible.

\section{References}

1. Araújo AR, Rosso N, Bedogni G, Tiribelli C, Bellentani S. Global epidemiology of non-alcoholic fatty liver disease/nonalcoholic steatohepatitis: What we need in the future. Liver Int. 2018;38(1):47-51. Available from: https://dx.doi.org/10.1111/ liv.13643.

2. Kalra S, Vithalani M, Gulati G, Kulkarni CM, Kadam Y, Pallivathukkal J, et al. Study of prevalence of nonalcoholic fatty liver disease (NAFLD) in type 2 diabetes patients in India (SPRINT). J Assoc Physicians India. 2013;61(7):448-453.

3. Cho Y, Tokuhara D, Morikawa H, Kuwae Y, Hayashi E, Hirose $\mathrm{M}$, et al. Transient Elastography-Based Liver Profiles in a Hospital-Based Pediatric Population in Japan. PLOS ONE. 2015;10(9):e0137239-e0137239. Available from: https://dx. doi.org/10.1371/journal.pone.0137239.

4. Day CP, James OFW. Steatohepatitis: A tale of two "hits"? Gastroenterol. 1998;114(4):842-845. Available from: https: //dx.doi.org/10.1016/s0016-5085(98)70599-2.

5. Tilg $\mathrm{H}$, Moschen AR. Evolution of inflammation in nonalcoholic fatty liver disease: The multiple parallel hits hypothesis. Hepatology. 2010;52(5):1836-1846. Available from: https://dx.doi.org/10.1002/hep.24001.

6. Yilmaz Y. Is nonalcoholic fatty liver disease the hepatic expression of the metabolic syndrome? World J Hepatol. 2012;4(12):332-334. Available from: https://dx.doi.org/10. 4254/wjh.v4.i12.332.
7. M M, JB H. Steatosis, Glycation and Liver Fibrosis in Patients with Diabetes. J Diabetes Metab. 2015;6(12):633633. Available from: https://dx.doi.org/10.4172/2155-6156. 1000633.

8. Listenberger LL, Han X, Lewis SE, Cases S, Farese RV, Ory DS, et al. Triglyceride accumulation protects against fatty acid-induced lipotoxicity. Proc Natl Acad Sci. 2003;100(6):3077-3082. Available from: https://dx.doi.org/10. 1073/pnas.0630588100.

9. Yamaguchi K, Yang L, McCall S, Huang J, Yu XX, Pandey SK, et al. Inhibiting triglyceride synthesis improves hepatic Steatosis but exacerbates liver damage and Fibrosis in obese mice with nonalcoholic steatohepatitis. Hepatology. 2007;45(6):13661374. Available from: https://doi.org/10.1002/hep.21655.

10. Wong VWS, Chan HLY, Elastography. Transient elastography. J Gastroenterol Hepatol. 2010;25(11):1726-1731. Available from: https://doi.org/10.1111/j.1440-1746.2010.06437.x.

11. Wong VWS, Vergniol J, Wong GLH, Foucher J, Chan HLY, Bail BL, et al. Diagnosis of fibrosis and cirrhosis using liver stiffness measurement in nonalcoholic fatty liver disease. Hepatology. 2010;51(2):454-462. Available from: https://dx. doi.org/10.1002/hep.23312.

12. Paradis V, Bedossa P. Definition and natural history of metabolic steatosis: histology and cellular aspects. Diabetes Metab . 2008;34(6):638-642. Available from: https://dx.doi. org/10.1016/s1262-3636(08)74598-1.

13. Tai FWD, Syn WK, Alazawi W. Practical approach to nonalcoholic fatty liver disease in patients with diabetes. Diabet Med. 2015;32(9):1121-1133. Available from: https://doi.org/ $10.1111 /$ dme. 12725 .

14. Younossi ZM, Gramlich T, Matteoni CA, Boparai N, McCullough AJ. Nonalcoholic fatty liver disease in patients with type 2 diabetes. Clin Gastroenterol Hepatol. 2004;2(3):262265. Available from: https://dx.doi.org/10.1016/s15423565(04)00014-x.

15. Jacqueminet S, Lebray P, Morra R, Munteanu M, Devers L, Messous D, et al. Screening for Liver Fibrosis by Using a Noninvasive Biomarker in Patients With Diabetes. Clin 
Gastroenterol Hepatol. 2008;6(7):828-831. Available from: https://dx.doi.org/10.1016/j.cgh.2008.03.005.

16. Marchesini G, Bugianesi E, Forlani G, Cerrelli F, Lenzi M, Manini R, et al. Nonalcoholic fatty liver, steatohepatitis, and the metabolic syndrome. Hepatology. 2003;37:917-923.

17. Friedrich-Rust M, Ong M, Martens S, Sarrazin C, Bojunga J, Zeuzem S, et al. Performance of Transient Elastography for the Staging of Liver Fibrosis: A Meta-Analysis. Gastroenterol. 2008;134(4):960-974. Available from: https://dx.doi.org/10. 1053/j.gastro.2008.01.034

18. Sasso M, Miette V, Sandrin L, Beaugrand M. The controlled attenuation parameter (CAP): A novel tool for the non-invasive evaluation of steatosis using Fibroscan ${ }^{\circledR}$. Clin Res Hepatol Gastroenterol. 2012;36(1):13-20. Available from: https://dx. doi.org/10.1016/j.clinre.2011.08.001.

19. Castéra L, Foucher J, Bernard PH, Carvalho F, Allaix D, Merrouche W, et al. Pitfalls of liver stiffness measurement: A 5-year prospective study of 13,369 examinations. Hepatology.
2010;51(3):828-835. Available from: https://dx.doi.org/10. $1002 /$ hep. 23425 .

Copyright: (C) the author(s), 2020. It is an open-access article distributed under the terms of the Creative Commons Attribution License (CC BY 4.0), which permits authors to retain ownership of the copyright for their content, and allow anyone to download, reuse, reprint, modify, distribute and/or copy the content as long as the original authors and source are cited.

How to cite this article: Sehgal R, Mittal J, Singh I. Correlation of Hepatic Steatosis with Hepatic Fibrosis in NAFLD Patients by Fibroscan. Asian J. Med. Res. 2020;9(3):1-7.

DOI: dx.doi.org/10.47009/ajmr.2020.9.3.ME1

Source of Support: Nil, Conflict of Interest: None declared. 